\title{
Stock Forecasting using Combination of Continuous Wavelet Transform and Neuro- Fuzzy Systems
}

\author{
M. Golchin \\ Department of Mathematics, Tehran North Branch, Islamic Azad University, Tehran, Iran \\ mahboobeh.golchin1@gmail.com
}

\begin{abstract}
Stock market forecasting is an important challenge for clients, so it attracts researchers to introduce new and reliable approaches to forecast this market. There are many approaches using wavelet decomposition to access details and approximation of stock market data and on the other hand, using neuro-fuzzy systems can be useful to overcome the uncertainty of data for forecasting. In this study, a hybrid approach will be introduced that is a combination of continues wavelet decomposition and neuro-fuzzy system to predict stock market data.

For this aim, first data is normalized between 0 to 1 and decomposed by continues wavelet decomposition and data of each sample time was used to forecast "n" sample forward and finally, this approach was evaluated.

For evaluating the performance of this approach, index of petroleum products of Tehran stock market between March 2015 to September 2019 was used and five steps of forecasting were considered. The best performance was $6.64 \times 10-5$ for one step forward forecasting and the worst was $4.74 \times 10-4$ for five steps forward forecasting.

Results showed that, increment of forecasting step causes decrement of forecasting precision however it is reliable.
\end{abstract}

Keywords-Forecasting, Neuro-Fuzzy, Wavelet transform

\section{INTRODUCTION}

Forecasting of stock market's features is an important challenge for companies and clients. So this problem attracts many researchers to introduce novel and efficient approaches to predict stock markets. On the other side this problem is extremely complex that makes the solving approaches hard[1], Stock markets are affected by many factors like economic variables, industry variables, company variables, psychological variables, and political variables [2] and all these factors can effect on stock market [3] therefore many combined approaches have been introduced [4] to develop automatic intelligent systems fits for stock market prediction.

There are many categories for stock market forecasting like financial time series forecasting using the number of input variables or multivariate analysis using effective variables. There are many approaches for financial time series prediction like autoregressive moving average (ARMA), the autoregressive integrated moving average (ARIMA), generalized autoregressive conditional heteroskedastic (GARCH) [5], smooth transition autoregressive model (STAR) [6], artificial neural network (ANN) [7], adaptive neuro-fuzzy system (ANFIS) [8, 9].

Combination of a fuzzy logic inference system (FIS) [10] with an artificial neural network results in a neuro-fuzzy controller that can be efficient for stock market prediction. Adaptive neuro fuzzy inference systems (ANFIS), are a usual approach in forecasting challenges [11]. This approach can predict most of complex patterns. But in fluctuating time series, it is better to fed these systems by approximations and details as input.

Wavelet transforms have been recently used in economic time series analysis and prediction [12] like oil price prediction [13] in combination with artificial neural networks [14]. Wavelet transform decomposes data into two sub series approximation and detail that can be used to softening data and improves forecasting accuracy.

In this work, the combination of continuous wavelet transform and neuro-fuzzy system would be evaluated for stock market prediction. For this aim we use this model for different steps of forecasting to evaluating the forecasting ability of this model.

\section{METHODS AND MATERIALS}

\section{A. Continuous Wavelet Transform}

Continuous wavelet transform (CWT) is a way to decompose time series in to wavelets and it is a good way to map the changing properties of non-stationary signals. CWT is a good approach for analysis financial time series. CWT of the time series is defined by:

$$
\begin{aligned}
& W(\tau, t)=\tau^{-1 / 2} \int_{-\infty}^{+\infty} p(x) \psi\left(\frac{x-t}{\tau}\right) d x \\
& \psi(t)=\frac{2}{\sqrt{3}} \pi^{-\frac{1}{4}}\left(1-t^{2}\right) e^{-\frac{t^{2}}{2}}
\end{aligned}
$$

where $\tau$ is the scaling factor, $t$ is the translating factor, $p(x)$ is time series and $\psi$ is the Mexican hat mother wavelet. The original time series $\mathrm{p}$ can be recovered from the wavelet representation $\mathrm{W}(\mathrm{p})$ by inverse transform [15]: 
$p(t)=\int_{-\infty}^{+\infty} \int_{0}^{+\infty} W(p)(\tau, t) t^{-\frac{5}{2}} \psi\left(\frac{t-x}{\tau}\right) d x d \tau$

The coefficients of the CWT have an effective amount of redundant information. So it would be usual to sample the coefficients in order to reduce redundancy. So in this study we decompose stock market time series into useful sets to fed to ANFIS to improve forecasting precision.

\section{B. ANFIS Model}

In this section, the ANFIS model would be introduced. to give us an idea of how the operation of ANFIS really is. For introducing simple, consider a fuzzy system with two rules [16]:

Rule 1 sif $x$ is $A_{1}$ and $y$ is $B_{1}$, then $f_{1}=p_{1}+p_{1} y_{1}+r_{1}$ Rule 2xif $x$ is $A_{2}$ and $y$ is $B_{2}$, then $f_{2}=p_{2}+p_{2} y_{2}+r_{2}$

In figure 1 the ANFIS reasoning mechanism and its architecture are illustrated. Nodes in the same layer have the same family of functions, as described below:

Layer 1: Nodes in this layer show the linguistic labels and their outputs are values of membership function. Changes in node's parameters would change the membership function's shape.

Layer 2: Each node in this layer calculates the firing power of each rule.

Layer 3: The ith node of this layer calculates the ratio of the firing strength of the ith rule to the sum of all the firing strength.

Layer 4: Nodes i in this layer have their function like rules.

Layer 5: The single node in this layer computes the overall output as the sum of all input signals.

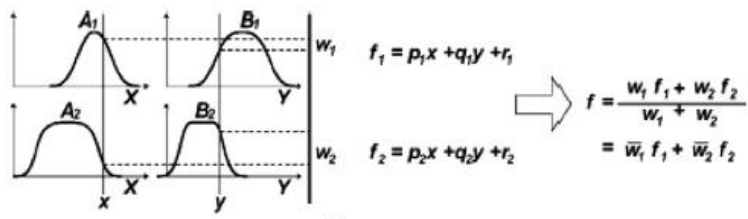

(a)

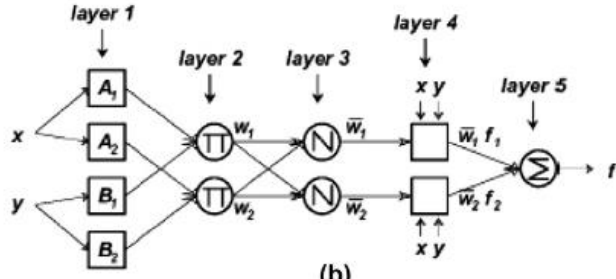

(b)

Fig 1. (a) Fuzzy model; (b) equivalent ANFIS architecture.

As yet, the adaptive network has been built which is equal to inference system, so it is called ANFIS and it would be a fuzzy inference system as an adaptive network.

\section{Combination Approach}

In this section the combination approach of CWT and ANFIS would be presented. This study follows these three step:
a) Data normalization
b) Continuous wavelet decomposition
c) Training neuro-fuzzy system for various prediction steps
d) Evaluating neuro-fuzzy system

At first step, data must be normalized. For this aim, the equation 4 will be used.

$$
N D=\frac{D-\min (D)}{\max (D)-\min (D)}
$$

While ND is normalized data and D is data. At second step, data will be decomposed by continuous wavelet decomposition to its scales and at next step, data would be divided in to train and test data and using the training data, neuro-fuzzy system must be trained. For this aim, each point of data can be used to predict $n$ step forward. For example, when the $\mathrm{i}^{\text {th }}$ sample point is the input of neuro-fuzzy system, the $i+n^{\text {th }}$ sample would be predicted. Finally, the trained neuro-fuzzy system would be used for testing and evaluating. The performance of this system will be computed by equation 5 .

$$
E=\frac{1}{N} \sum_{i=1}^{N}(N D-\widehat{N D})^{2}
$$

Where $\widehat{N D}$ is predicted normalized data.

\section{RESULTS}

For simulating and evaluating this approach, the index of petroleum product of Tehran stock market since March 2015 to September 2019 is used and normalized this time series contained 1249 samples. The time series of this data and its normalized data is illustrated in figure 2 .
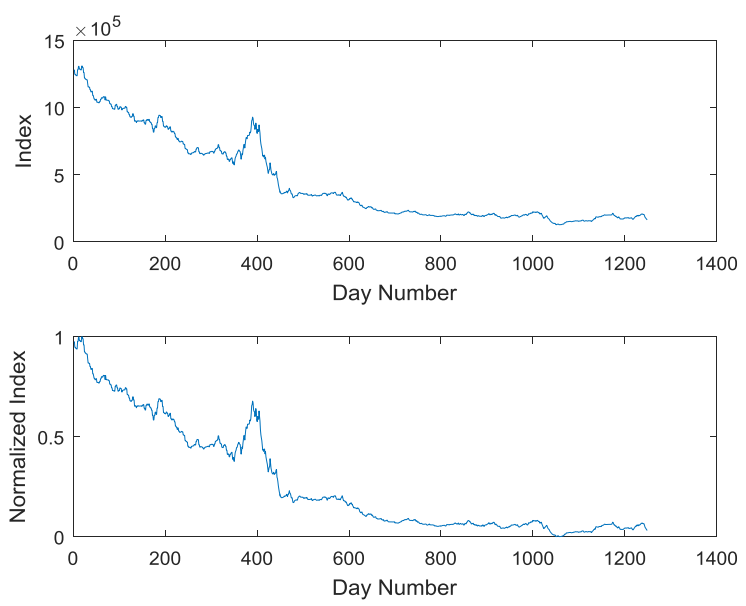

Fig 2. Stock market data since March 2015 to September 2015 and its normalized form

At next step data decomposed by continues wavelet decomposition with sym 2 mother wavelet into 4 scales as figure 3 . 


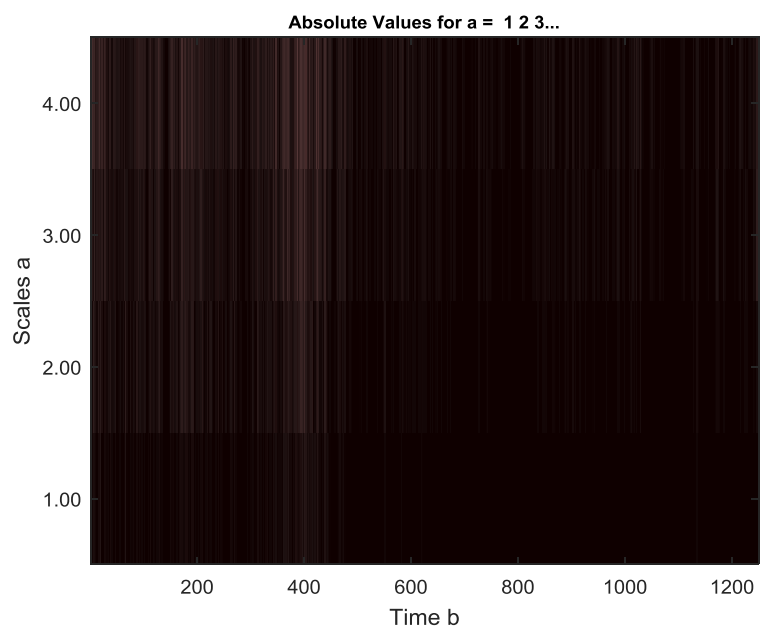

Fig 3. Continues wavelet decomposition of normalized data into 4 scales

Then data was divvied into training data (1000 samples) and test data (249 samples). Next scale of each sample time was used for forecasting 1 to 5 sample forward. The used neurofuzzy system used Sugeno fuzzy decision machine and 3 gaussian membership function as fuzzifier and gravity center as defuzzifier. A comparision between one step forecasted data and real data is illustrated in figure 4 and the performance for all steps was computed and illustrated as table 1 .

(a)

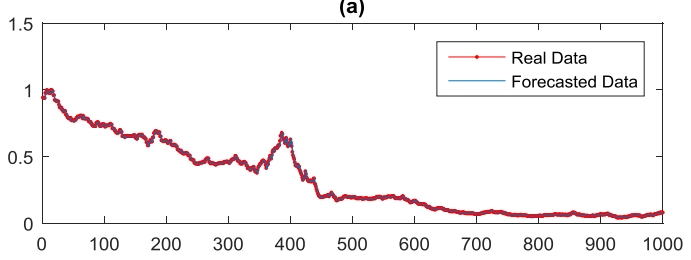

(b)

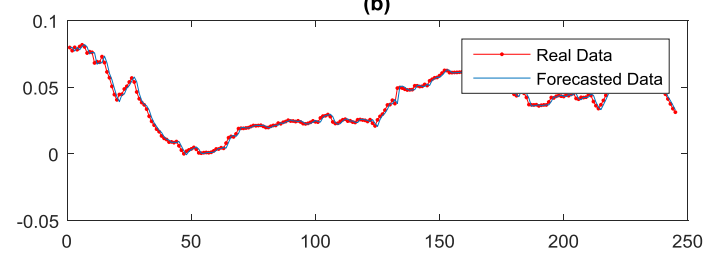

Fig 4. Comparison between real data and one step forecasted data (a) for train data and (b) for test data

Table 1. Performance of test and train for different forecasting steps

\begin{tabular}{|c|c|c|}
\hline Step Number & $\begin{array}{c}\text { Train } \\
\text { Performance }\end{array}$ & Test Performance \\
\hline 1 & $6.64 \times 10^{-5}$ & $6.23 \times 10^{-5}$ \\
\hline 2 & $1.48 \times 10^{-4}$ & $1.72 \times 10^{-5}$ \\
\hline 3 & $2.47 \times 10^{-4}$ & $3.13 \times 10^{-5}$ \\
\hline 4 & $3.63 \times 10^{-4}$ & $4.74 \times 10^{-5}$ \\
\hline 5 & $4.74 \times 10^{-4}$ & $6.57 \times 10^{-5}$ \\
\hline
\end{tabular}

\section{CONCLUSION}

In this study a new approach is introduced to forecast stock market indexes that was a combination of neuro-fuzzy system and continues wavelet decomposition. For this aim there was four steps. First data was normalized between zero to one and decomposed into its scales using continues wavelet decomposition, then neuro-fuzzy system was trained and evaluated for various forecasting steps. Results showed that, increment of forecasting step causes decrement of forecasting precision however it is reliable.

\section{REFERENCES}

G. J. Deboeck, Trading on the edge: neural, genetic, and fuzzy systems for chaotic financial markets. John Wiley \& Sons, 1994. J.-Z. Wang, J.-J. Wang, Z.-G. Zhang, and S.-P. Guo, "Forecasting stock indices with back propagation neural network," Expert Systems with Applications, vol. 38, no. 11, pp. 14346-14355, 2011.

J. Yao, C. L. Tan, and H.-L. Poh, "Neural networks for technical analysis: a study on KLCI," International journal of theoretical and applied finance, vol. 2, no. 02, pp. 221-241, 1999.

G. S. Atsalakis and K. P. Valavanis, "Surveying stock market forecasting techniques-Part II: Soft computing methods," Expert Systems with Applications, vol. 36, no. 3, pp. 5932-5941, 2009. P. H. Franses and H. Ghijsels, "Additive outliers, GARCH and forecasting volatility," International Journal of Forecasting, vol. 15 , no. 1, pp. 1-9, 1999

N. Sarantis, "Nonlinearities, cyclical behaviour and predictability in stock markets: international evidence," International Journal of Forecasting, vol. 17, no. 3, pp. 459-482, 2001.

X. Zhong and D. Enke, "Forecasting daily stock market return using dimensionality reduction," Expert Systems with Applications, vol. 67, pp. 126-139, 2017.

M. S. C. Lah, N. Arbaiy, and R. Efendi, "Stock Market Forecasting Model Based on AR (1) with Adjusted Triangular Fuzzy Number Using Standard Deviation Approach for ASEAN Countries," in Intelligent and Interactive Computing: Springer, 2019, pp. 103-114.

N. Srinivasan and C. Lakshmi, "Stock Price Prediction Using Fuzzy Time-Series Population Based Gravity Search Algorithm," International Journal of Software Innovation (IJSI), vol. 7, no. 2, pp. 50-64, 2019.

S. Guillaume and B. Charnomordic, "Learning interpretable fuzzy inference systems with FisPro," Information Sciences, vol. 181, no. 20, pp. 4409-4427, 2011.

G. Makridou, G. S. Atsalakis, C. Zopounidis, and K. Andriosopoulos, "Gold price forecasting with a neuro-fuzzy-based inference system," International Journal of Financial Engineering and Risk Management 2, vol. 1, no. 1, pp. 35-54, 2013. 
[12] S. K. Aggarwal, L. M. Saini, and A. Kumar, "Electricity price forecasting in Ontario electricity market using wavelet transform in artificial neural network based model," International Journal of Control, Automation, and Systems, vol. 6, no. 5, pp. 639-650, 2008.

[13] Z. Luo et al., "Oil Price Forecasting Using Supervised GANs with Continuous Wavelet Transform Features," in 2018 24th International Conference on Pattern Recognition (ICPR), 2018, pp. 830-835: IEEE.

[14] R. Jammazi and C. Aloui, "Crude oil price forecasting: Experimental evidence from wavelet decomposition and neural network modeling," Energy Economics, vol. 34, no. 3, pp. 828841, 2012.

[15] G. Peyré, A wavelet tour of signal processing: the sparse way. Academic Press, 2009

[16] P. Melin, J. Soto, O. Castillo, and J. Soria, "A new approach for time series prediction using ensembles of ANFIS models," Expert Systems with Applications, vol. 39, no. 3, pp. 3494-3506, 2012.
Creative Commons Attribution License 4.0
(Attribution 4.0 International, CC BY 4.0)

This article is published under the terms of the Creative Commons Attribution License 4.0

https://creativecommons.org/licenses/by/4.0/deed.en_US 